\title{
Research on Hybrid Simulation Test Method for Cooperative Deformation of Membrane and Frame Composite Structures
}

\author{
Yu Yangchun ${ }^{1}$, Xu Xiaochen ${ }^{2}$, Xu Zhihong ${ }^{1}$ \\ ${ }^{1}$ Science School, Nanjing University of Science and Technology, Nanjing, China \\ ${ }^{2}$ Nanjing Institute of Electronic Technology, Nanjing, China
}

Email address:

yuyangchun77@126.com (Yu Yangchun),xuzh@njust.edu.cn (Xu Zhihong)

\section{To cite this article:}

Yu Yangchun, Xu Xiaochen, Xu Zhihong. Research on Hybrid Simulation Test Method for Cooperative Deformation of Membrane and Frame Composite Structures. Science Discovery. Vol. 7, No. 5, 2019, pp. 359-364. doi: 10.11648/j.sd.20190705.27

Received: October 26, 2019; Accepted: November 25, 2019; Published: December 3, 2019

\begin{abstract}
In this paper, based on the joint laboratory of Mechanics Test Center of Nanjing University of Science and Technology and Walter+Bai, the PTFE coated fabric film material is taken as the test object, and the A/D signal exchange between the numerical substructure and the experimental substructure is realized by PMI serial port program, and the hybrid simulation static test is carried out. The phenomenon that the supporting structure of the frame is deformed due to the interaction in the tension process of the membrane structure is simulated, and then the unloading effect on the membrane surface is produced. The test results show that the communication performance of PMI serial port program is good, and the time consumption produced by signal exchange process can be ignored. The accuracy and stability of the hybrid simulation test system are verified by comparing the mixed simulation test results with the pure numerical simulation theoretical solution. Through the comprehensive analysis of the test data such as stress, strain, displacement, loading and unloading times, some suggestions are put forward for the design of membrane and frame composite structure.
\end{abstract}

Keywords: PMI, Sub-structure, Hybrid Simulation, Unloading Effect, Composite Structure

\section{针对膜与框架组合结构协同变形的混合仿真试验方法研究}

\section{俞阳椿 ${ }^{1}$, 徐晓晨 $^{2}$, 徐志洪 $^{1}$}

1 南京理工大学理学院, 南京, 中国

2南京电子技术研究所, 南京, 中国

\section{邮箱}

yuyangchun77@126.com(俞阳椿), xuzh@njust.edu.cn(徐志洪)

摘要: 本文依托南京理工大学力学试验中心与Walter+Bai联合实验室, 以PTFE涂层织物膜材为试验对象, 借助PMI串 口程序实现数值子结构与试验子结构之间的 $\mathrm{A} / \mathrm{D}$ 信号交换, 进行了混合仿真静力试验。试验模拟了膜结构在张拉过程 中, 由于相互作用, 使与之相连的框架支承结构发生形变, 进而对膜面产生卸载效应的现象。试验结果显示: PMI串 口程序通讯性能良好，信号交换过程产生的时间消耗可忽略不计; 通过混合仿真试验结果与纯数值模拟理论解的对比 分析, 验证了混合仿真试验系统的精确性和稳定性; 通过对试验所得应力、应变、位移、加卸载次数等数据综合分析, 为膜与框架组合结构的设计提出建议。 
关键词: PMI, 子结构, 混合仿真, 卸载效应, 组合结构

\section{1. 引言}

膜结构因其质轻、透光、建筑表现力强等优点, 被 广泛应用于现代建筑中。膜结构按照产生预张力的方式 不同可分为三类: 充气式膜结构、张拉式膜结构以及骨 架支承式膜结构。张拉膜结构是将高强薄膜材料通过张 拉固定的方式使其内部产生预应力以形成某种空间结构 形式, 作为覆盖结构, 并承受一定的外荷载作用 [1], 如 图1。张拉膜结构是最能展现膜结构精神的构造形式, 结 构性能强, 且具有丰富的表现力。膜结构体系在张拉成 形的过程中经历了三个状态, 分别是: 无预应力的松他 机构状态、逐步施加预应力过程到结构找形状态、预应 力张拉完成的结构成形状态 [2]。逐步施加预应力的过程 就是膜结构刚度不断发生变化, 同时结构形态和受力特 性也不断发生变化的过程。在这个过程中, 由于膜与以 框架为代表的支承体系间具有相互作用, 框架受力后逐 渐产生变形, 如图2。边界条件的改变使得膜结构原有的 平衡态被打破, 膜与框架产生协同变形, 进而引起膜内 应力重分布, 以达到新的平衡态。张拉膜结构最终的位 移、应力水平是整个张拉过程各个阶段位移、应力不断 变化和累积的结果 [3], 因此框架的变形必然对结构的最 终成形状态产生影响。

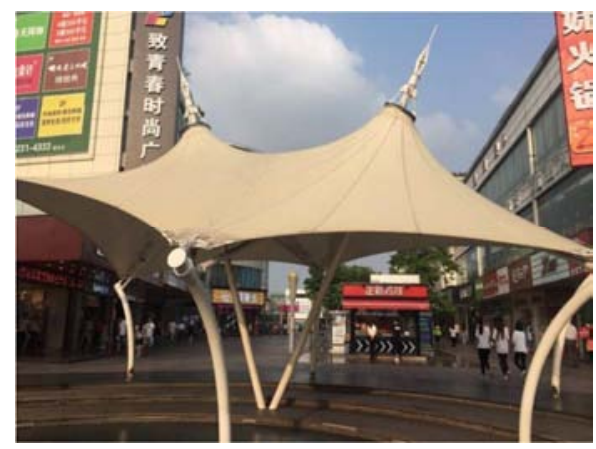

图1 张拉膜。

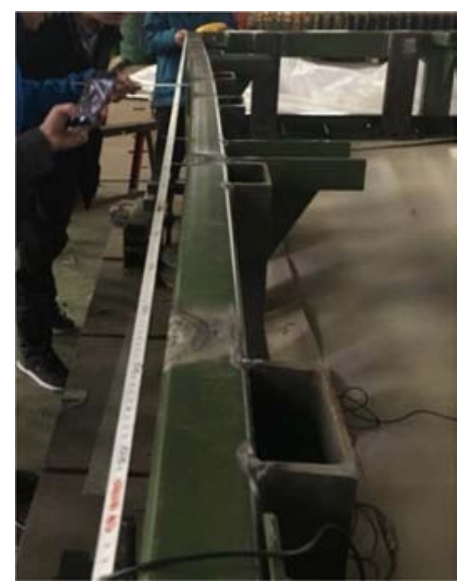

图2 张拉膜试验后框架明显变形。
目前, 在进行张拉膜结构设计时并不太注重框架支承 体系的变形分析，而是简单地增加支承体系的材料用量以 增大支承体系的刚度和支撑能力。这是因为膜材是强非线 性材料, 其本构关系受到多种因素影响且难以采用 ANSYS等有限元软件进行数值模拟 [4], 无法在设计过程 中高效、准确地预测膜与框架之间的协同变形。单纯增加 支承体系材料用量的做法不但会造成材料的盲目浪费，在 某些对设备或构筑物有重量限制的场合如军用设施中, 也 无法满足设计要求。

混合仿真试验方法是一种子结构技术 [5], 将全结构 划分为试验子结构和数值 (计算) 子结构, 其中非线性 比较突出的部分作为试验子结构在实验室进行物理试 验, 其余部分作为数值子结构通过计算机软件进行数值 计算, 两者通过通讯串口程序实现 $\mathrm{A} / \mathrm{D}$ (analog/digital) 信号的实时传递, 从而达到降低试验难度、提升试验效 率以及减少试验成本的目的 [6]。国内外已有众多学者专 家对混合仿真这一试验方法进行了深入研究。Shao X向 读者阐释了混合仿真试验方法的思想原理, 并借助 MATLAB\&Simulink建立了数值子结构, 与试验子结构 形成闭合循环试验体系 [7]。UC Berkeley（美国加州大 学伯克利分校) 借助OpenSees数值模拟软件, 开发了 OpenFresco接口程序, 并利用MTS (美特斯) 液压控制 系统，建立了一套完整的混合仿真试验体系 $[8]$ 。本文基 于数值计算软件MATLAB和瑞士 $\mathrm{W}+\mathrm{B}$ 试验设备公司生 产的LFV-10型电液伺服多功能试验机, 借助南京理工大 学力学实验中心自行开发的PMI通讯串口程序 [9-11], 对膜与框架组合结构进行混合仿真试验, 研究其协同变 形关系以及框架变形对膜的卸载效应, 并为张拉膜结构 支承体系设计提出建议。

\section{2. 混合仿真试验体系构建}

膜在张拉过程中其自身内力和刚度不断发生变化, 与 之相连的框架支承结构由于膜与框架的相互作用也发生 相应变形, 如图3。在膜结构施工中, 无法通过一次张拉 就使膜达到指定预张力, 因此需要进行多次张拉, 而每一 次张拉固定都会以协同作用力的形式作用在框架上使其 产生变形, 框架变形使得膜的伸长量减小即膜面张力减小, 并达到新的平衡态。由于膜的刚度是随着张拉次数的增加 而不断变化的, 因而很难通过有限元软件计算得到最终位 移。子结构混合仿真试验将膜作为试验子结构, 从每次张 拉打破原有平衡状态到膜与框架协同变形达成新的平衡 状态的过程作为一个试验子步, 在张拉的瞬间由于时间很 短, 可将膜（试验子结构）的刚度视为固定[12], 从而进 行静力计算。

混合仿真试验系统由三部分组成, 包括电液伺服加载 系统、由MATLAB编写的数值计算方法和二者之间的通讯 串口程序PMI, 具体分为LFV-10型电液伺服多功能试验机、 PCS8000 控制器、DION7 控制软件以及 PMI 
(PCS8000-MATLAB-Interface) 通讯接口程序。LFV-10 型电液伺服多功能试验机外形简图如图4, 规格参数如表1 所示[13]。DION7控制软件是Walter+Bai公司开发的通用 型配套软件, 可与该公司旗下的多款试验设备搭配使用, 搭载DION7控制软件的试验计算机借助串口（COM接口） 与PCS8000控制器相连, 实现计算机与控制器的双向通信 和A/D信号转换。DION7控制软件主要有两个功能模块: EASY和FPI。EASY模块主要针对信号单一的试验, 对结 构或构件进行疲劳拉伸、压缩以及正余弦加载, 操作简单, 可试验性强[14], 但不适用于复杂信号的模拟。FPI模块是 可编辑模块, 需自主编辑试验流程图（如图5), 能够完 成多信号甚至复杂信号的输入。通过使用FPI模式对加载 方式进行二次开发, 实现通讯接口程序PMI和PCS8000控 制器的数据传递。其中PMI通讯接口程序是基于 $\mathrm{C}++$ 编写 的, 因为 $\mathrm{C}++$ 语言相较于其他计算机语言计算效率较高, 可大大减小软件的响应时间, 从而有效减小试验过程中由 计算软件产生的时滞误差[15]。MATLAB编写的数值计算 方法以 $\mathrm{m}$ 文件的格式内嵌在PMI程序中, PMI通过调用 $\mathrm{m}$ 文 件实现数值子结构与试验子结构之间的信号传递。在试验 进行过程中, PMI的记录模块会对试验机反馈的力、位移、 速度、加速度等信息进行实时监控和记录, 并在试验结束 后以 txt文本格式输出。混合仿真系统组成架构如图6所示。

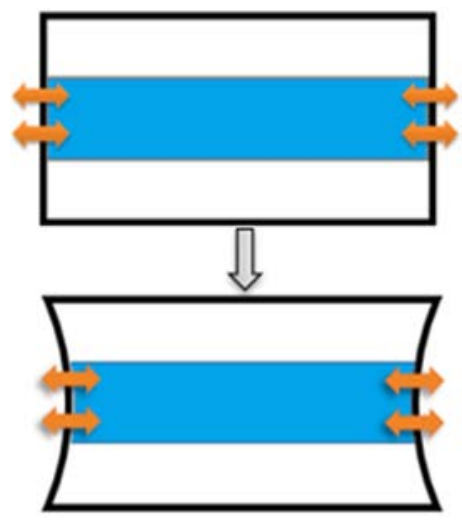

图3 膜与框架相互作用示意图。

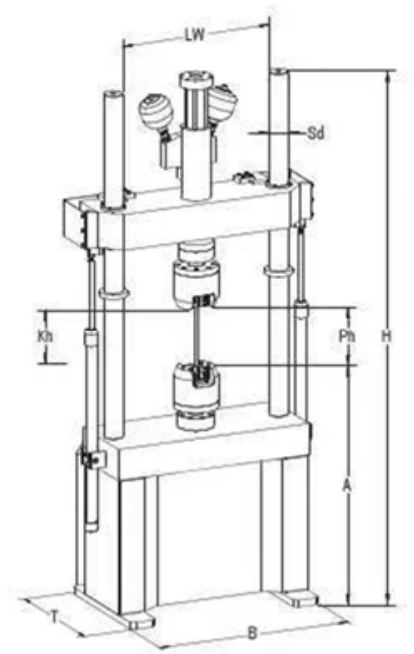

图4 LFV-10型电液伺服多功能试验机外形简图。
表1 LFV-10型电液伺服多功能试验机规格参数。

\begin{tabular}{lll}
\hline Max. Test Load Static & $\mathrm{kN}$ & \pm 10 \\
Max. Test Load Dynamic & $\mathrm{kN}$ & \pm 8 \\
Piston Stroke (Kh) & $\mathrm{mm}$ & \pm 50 \\
Max. Distance betw. Grips (Ph) & $\mathrm{mm}$ & 1000 \\
Distance betw. Columns (LW) & $\mathrm{mm}$ & 800 \\
Column Diameter (Sd) & $\mathrm{mm}$ & 80 \\
Working Height (A) & $\mathrm{mm}$ & 750 \\
Width (B) & $\mathrm{mm}$ & 1220 \\
Depth (T) & $\mathrm{mm}$ & 1000 \\
Height (H) & $\mathrm{mm}$ & 3330 \\
Weight (without Grips) & $\mathrm{kg}$ & 1800 \\
Frame Stiffness & $\mathrm{kN} / \mathrm{mm}$ & 300 \\
\hline
\end{tabular}

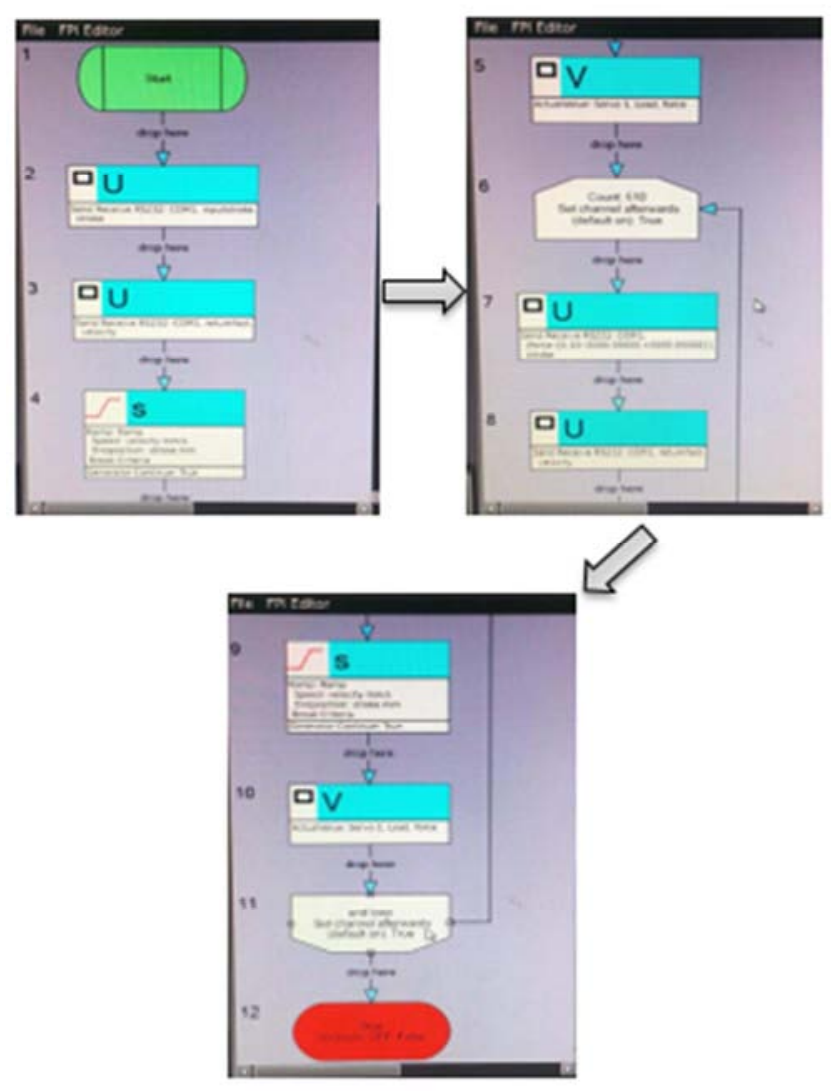

图5 混合仿真试验自定义流程图。

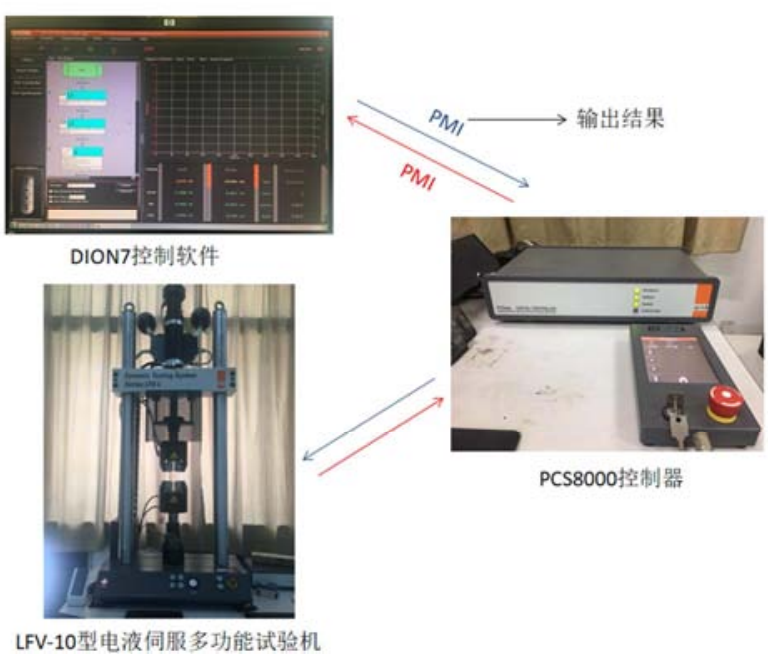

图6 混合仿真系统组成架构图。 


\section{3. 混合仿真静力试验与单轴循环拉伸试验}

\section{1. 混合仿真静力试验}

本文拟定 $1500 \mathrm{~mm} \times 1500 \mathrm{~mm}$ 矩形框架, 对尺寸为 $1500 \mathrm{~mm} \times 500 \mathrm{~mm}$ 的PTFE涂层织物膜材进行张拉固定。框 架材料采用碳钢, 在小变形条件下满足胡克定律, 弹性模 量 $206 \mathrm{Gpa}$, 横截面形式为 $50 \mathrm{~mm} \times 50 \mathrm{~mm}$ 矩形截面。张拉方 式为“分段式”张拉, 即一边张拉一边固定, 直至膜面应力 达到指定预张力或者膜材张拉伸长量达到预设值。在混合 仿真试验中, 以每个子步张拉特定位移的方式模拟膜的张 拉过程。从上个平衡态到新的平衡态框架的变形量为当前 子步的框架变形量, 每个子步框架变形量的叠加即为整个 张拉过程框架的最终变形量。在膜结构设计中, 为安全起 见, 取框架变形最大处即框架中点处的变形量为目标参数。 在混合仿真系统的数值子结构中, 判断每个试验子步膜面 达到新平衡态的条件是: 膜与框架产生协同变形后, 膜与 框架衔接处两侧 (膜和框架) 力平衡, 即试验子结构反馈 的力值和数值子结构计算所得力值相等。由于试验机尺寸 限制, 仅针对小型试件进行混合仿真试验, 为了减小尺寸 效应的影响, 将试验子结构的膜材尺寸和数值子结构的框 架尺寸同步缩小为原尺寸的 $1 / 10$, 即试验膜材尺寸为 $150 \mathrm{~mm} \times 50 \mathrm{~mm}$, 框架尺寸为 $150 \mathrm{~mm} \times 150 \mathrm{~mm}$ 。试验膜材需 沿着纱线编织的纹路裁剪得到, 其中经向长度为 $150 \mathrm{~mm}$, 纬向长度为 $50 \mathrm{~mm}$, 如图7所示。膜材经向两端保留冗余部 分用作夹持端, 夹持方式为卷曲式夹持, 试验过程未发生 明显滑移。
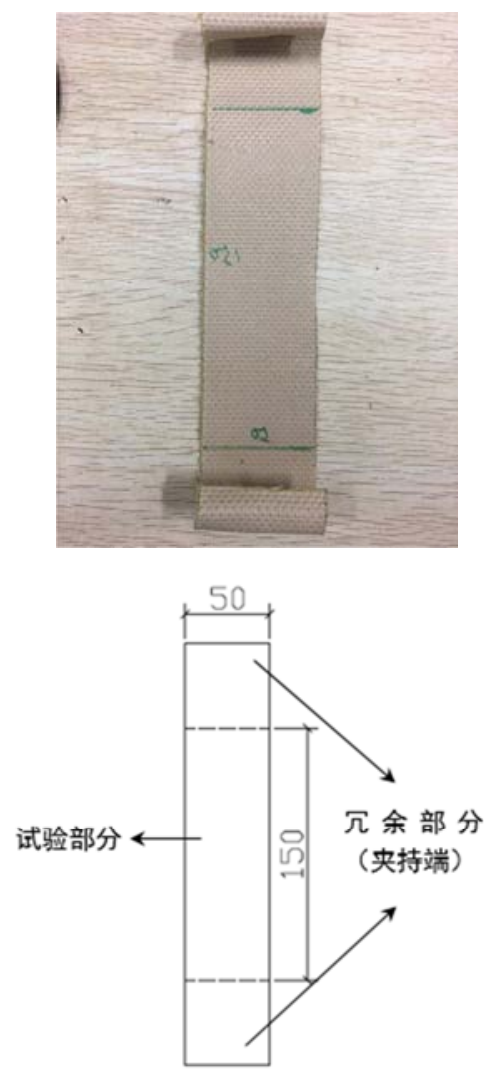

图7 试验膜材及夹持端。
混合仿真试验每个子步预张拉 $0.1 \mathrm{~mm}$, 并在每个子步 中将当前子步框架变形量反馈给试验膜材, 当膜材最终张 拉伸长量达到 $7.5 \mathrm{~mm}$ 即应变达到 $5 \%$ 时试验终止。PMI将每 个子步预张拉后的膜材应力应变值和每个子步将框架变 形反馈给膜材并达到新平衡态后的膜材应力应变值以数 列的形式通过 $\mathrm{txt}$ 文件输出。输出结果如图8所示, 曲线为 每个子步将框架变形反馈给膜材并达到新平衡态后的膜 材应力应变曲线。

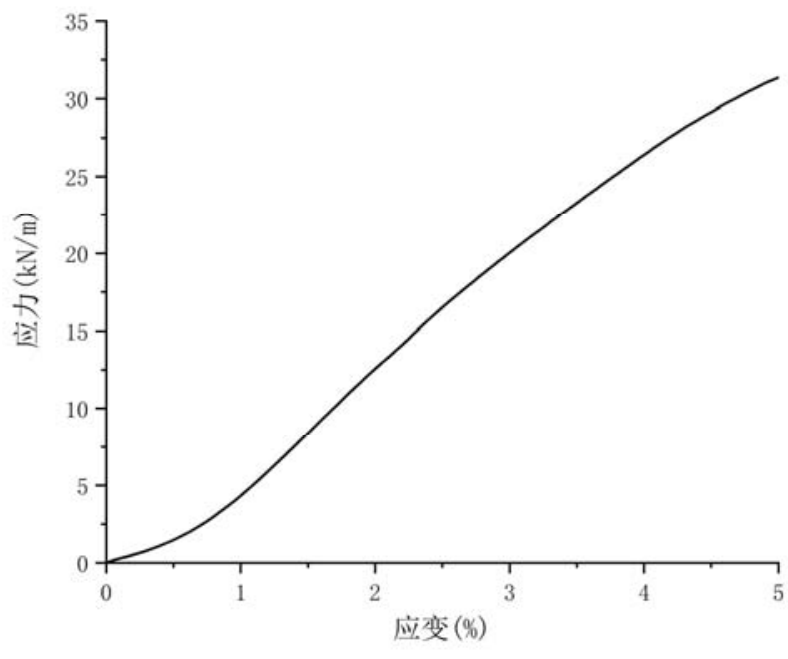

图8 混合仿真试验结果。

\section{2. 单轴循环拉伸试验}

采用与上述试验膜材相同的裁剪方法得到另外 4 个试 件, 其中 3 个试件进行单轴循环拉伸试验, 1 个试件进行单 轴单调拉伸试验。根据《膜结构检测技术规程》 DG/TJ08-2019-2007规定, 试验温度为 $23 \pm 2^{\circ} \mathrm{C}$, 相对湿度 为 $65 \% \pm 3 \%$, 试验仪器如图9所示。试验过程严格控制加、 卸载速率不发生改变, 控制加、卸载速率为 $2 \mathrm{~mm} / \mathrm{min}$ 。试 验可得 3 个试件的单轴循环拉伸应力-应变曲线和 1 个试件 的单轴单调拉伸应力-应变曲线, 如图10所示。

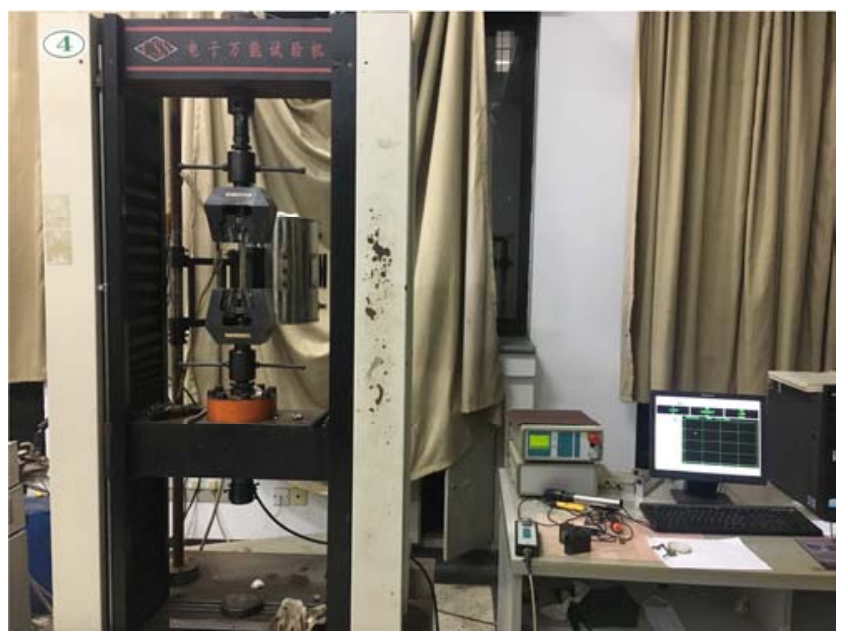

图9 电子万能试验机。 


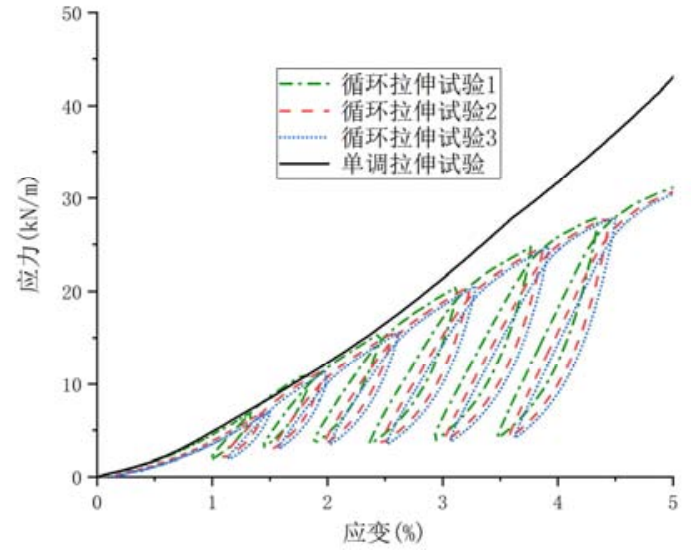

图10 单轴循环拉伸和单调拉伸试验曲线。

由图10可以看出, 3 条循环拉伸曲线趋势一致, 说明 试件本身的误差很小，膜材尺寸、裁剪方法控制到位。另 外, 3 条循环拉伸曲线与单调拉伸曲线在第 1 次卸载前吻合 度很好, 在第4次卸载前单调拉伸曲线对循环拉伸曲线大 致形成包络。随着加、卸载次数的增加, 单调拉伸曲线与 循环拉伸曲线逐渐分开, 且趋势越来越明显。循环拉伸和 单调拉伸的对比试验证明: 1. 循环拉伸试验的首次加载弹 性模量与同等应力范围内单调拉伸的弹性模量相同; 2 . 循 环拉伸过程存在能量的耗散 [16], 循环次数越多, 能量耗 散越多, 曲线上升趋势与单调拉伸曲线上升趋势差异越大。 3. 膜材拉伸力学性能受拉伸方式影响较大, 因此在研究膜 与框架组合结构协同变形时应考虑到框架变形对膜面的 卸载效应, 不能简单套用膜材单调拉伸本构模型。表 2 是 应变达到 $5 \%$ 时, 循环拉伸和单调拉伸的 4 个试验膜材各自 达到的应力值。

表2 应变达到 0.05 时各试件的应力值。

\begin{tabular}{lllll}
\hline 试件 & 循环拉伸1 & 循环拉伸2 & 循环拉伸3 & 单调拉伸 \\
\hline 应力值 $\mathrm{kN} / \mathrm{m}$ & 31.14 & 30.69 & 30.46 & 43.09 \\
\hline
\end{tabular}

\section{3. 试验结果分析}

将图8和图10, 即混合仿真试验所得每个子步预张拉 后的膜材应力应变曲线、循环拉伸应力应变曲线和单调拉 伸应力应变曲线绘制在同一张图中，如图11所示。

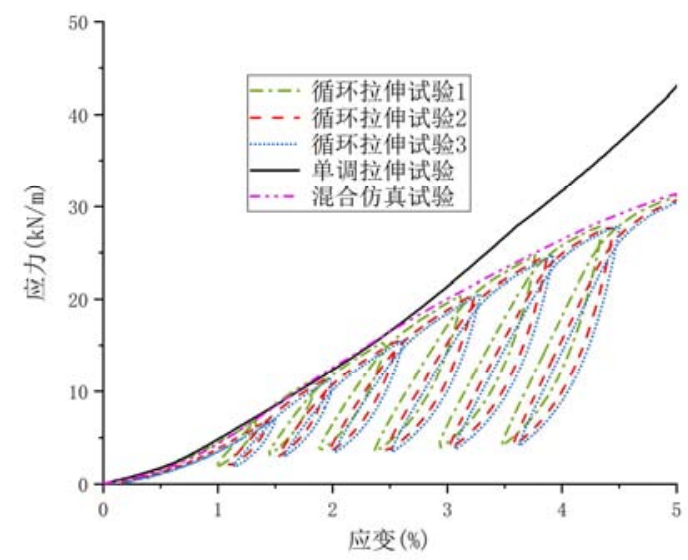

图11 混合仿真、循环拉伸和单调拉伸应力应变曲线。
图11显示, 混合仿真试验所得每个子步预张拉后的膜 材应力应变曲线对循环拉伸应力应变曲线完美包络, 说明 针对膜与框架组合结构协同变形关系的混合仿真试验结 果很好地描绘了膜材在循环拉伸作用下力学性能的变化 过程, 证明了张拉膜结构在膜材张拉过程中由于以框架为 代表的支承体系发生变形对膜材内力的影响, 同时也验证 了混合仿真试验方法在静力学问题研究上的有效性和准 确性。从图中可以看出, 当应变值达到 $5 \%$ 时, 单调拉伸 试验膜材的应力值为 $43.09 \mathrm{kN} / \mathrm{m}$, 混合仿真试验膜材的应 力值为 $31.35 \mathrm{kN} / \mathrm{m}$, 两者相差 $27.25 \%$ 。根据应变能公式

$$
\mathrm{W}=\int_{0}^{\varepsilon} \delta(\varepsilon) d \varepsilon
$$

计算可得混合仿真试验结果相较单调拉伸试验结果 应变能减少了 $2201.25 \mathrm{~J}$, 即张拉过程中由于框架变形使得 膜材经历多次加、卸载循环后在应变达到 $5 \%$ 这一指标点 时消耗了 2201.25J的能量。混合仿真结果与单调拉伸结果 的巨大差异证明了张拉膜结构中膜材和框架的一体性, 即 框架变形会对膜材内力产生巨大影响, 膜结构设计者在设 计计算和分析过程中不应忽视框架的变形及膜与框架之 间的协同关系。

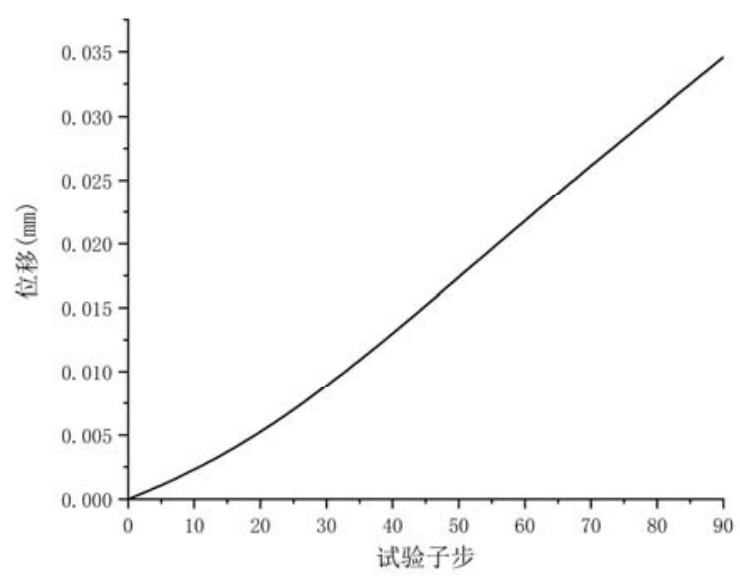

a 各试验子步框架中点变形量

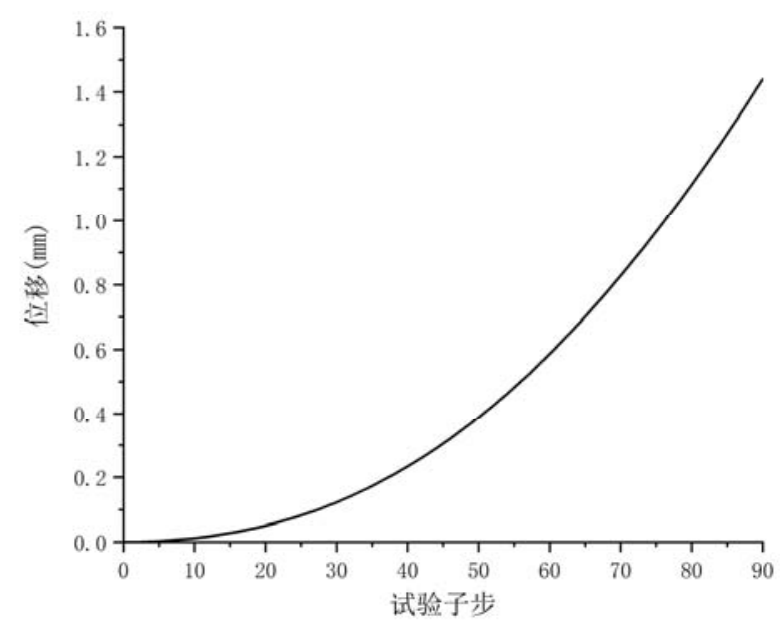

$\mathrm{b}$ 框架中点总变形随试验子步增长曲线

图12 框架中点变形曲线。 
图12给出了框架受力边中点即变形最大处的位移变 化曲线。其中, 图12a为每个试验子步框架中点变形量, 图12b为框架中点总变形量曲线。由图可知, 在膜材最终 变形量达到 $7.5 \mathrm{~mm}$ 的 90 个试验子步中, 框架中点每个试验 子步的变形量逐渐增大, 这是由于膜材在张拉伸长过程中 抗拉刚度逐渐增大, 膜材对框架的作用力也逐渐增大。框 架中点最终变形量为 $1.44 \mathrm{~mm}$ 。

\section{4. 结语}

本文以膜与框架组合结构为对象, 采用混合仿真试验 方法, 对其协同变形关系进行了研究。通过混合仿真试验 结果与单轴循环拉伸试验结果对比, 证明膜与框架组合结 构在张拉过程的力学性能符合膜材的循环拉伸力学性能; 通过混合仿真试验结果与单轴单调拉伸试验结果对比, 表 明膜与框架组合结构在张拉过程中存在着能量损耗, 并给 出能量损失计算方法; 混合仿真试验结果验证了混合仿真 试验系统的稳定性和可靠性, 为后续将混合仿真试验方法 推广到更多含非线性构件组合结构的静力试验中奠定了 基础。

本文研究的针对膜与框架组合结构协同变形的混合 仿真试验方法可通过改变数值子结构中 $\mathrm{m}$ 文件的框架尺 寸、截面形式以及材料弹性模量来预测框架变形量, 为组 合结构框架支承部分的设计提供依据。

\section{参考文献}

[1] 蒋泽峰. 张拉膜结构的全过程设计及找形分析 [D]. 北京: 清华大学, 2000.

[2] 段有恒. 索膜结构张拉及加载全过程仿真分析研究[D]. 北 京: 北京交通大学, 2012 .

[3] 邱鹏. 空间网格结构滑移法施工全过程分析方法及若干关 键问题的研究 [D]. 杭州: 浙江大学, 2006 .
[4] 李永祥. 考虑弹性支承的整体张拉膜结构力学性能研究 [D]. 兰州: 兰州理工大学, 2008 .

[5] 郝海莉. 基于MTS试验系统的空间SRT混合仿真试验方法 研究[D]. 西安: 西安建筑科技大学, 2015.

[6] 章红梅, 曾松, 段元锋, 等. 实时混合试验方法研究与应 用综述 [J]. 结构工程师, 2015,31(1):207-212.

[7] Shao X, Enyant G. Development of a versatile hybrid testing system for seismic experimentation [J]. Experimental Techniques, 2015, 38 (6): 44-60.

[8] Schellenberg A, Kim H K, Takahashi Y, et al. OpenFresco command language manual $[\mathrm{M}]$. Berkeley, US: The Regents of the University of California, 2009.

[9] 张祁, 董成, 徐志洪. 含粘弹性阻尼器结构的实时混合仿 真研究[J]. 南京理工大学学报, 2017, 41(5):623-626+633.

[10] 董成. 含速度相关型阻尼器的结构混合仿真研究[D]. 南京: 南京理工大学, 2017.

[11] 曹志威, 徐志洪, 张祁, 等. 带粘弹性阻尼器的斜拉索动 力混合仿真[J]. 华北地震科学, 2017,35(2):66-69.

[12] 刘振邦, 张祁, 徐志洪. 含非线性拉杆的拱结构的混合仿 真研究 [J]. 南京理工大学学报, 2018, 42(5):547-551.

[13] Walter+Bai公司生产的LFV-10型电液伺服多功能试验机说 明书。

[14] 张祁. 带粘弹性阻尼器框架结构的混合仿真若干问题的研 究[D]. 南京: 南京理工大学, 2018.

[15] Ge Yang, Bin Wu, Ge Ou, Zhen Wang, Shirley Dyke. HyTest: Platform for Structural Hybrid Simulations with Finite Element Model Updating [J]. Advances in Engineering Software, 2017, 112: 200-210.

[16] 陈建稳, 陈务军, 张大旭. PVDF/PES涂层织物循环拉伸力 学性能及弹性模量 [J]. 华南理工大学学报 (自然科学 版）,2013,41(6):69-76. 